susceptibility to ceftriaxone, spectinomycin, ciprofloxacin and tetracycline were tested using agar dilution method, according to the recommendations from CLSI. NG-MAST was performed for molecular epidemiology and full-length por $B$ sequences were used for phylogenetic analysis.

Results All (100\%) isolates were resistant to ciprofloxacin, tetracycline, and $41.6 \%$ produced $\beta$-lactamase. According to the CLSI breakpoints, all $(100 \%)$ isolates were susceptible to spectinomycin $(\mathrm{S}<=$ $32 \mu \mathrm{g} / \mathrm{ml}$ ) and $99.7 \%$ to ceftriaxone $(\mathrm{S} \leq 0.25 \mu \mathrm{g} / \mathrm{ml})$. However, using the European breakpoints $5.2 \%$ of the isolates were resistant to ceftriaxone (EUCAST, $S \leq 0.125 \mu \mathrm{g} / \mathrm{ml}$ ). The most prevalent NG-MAST clusters in 2007 included ST568 $(\mathrm{n}=13)$, ST270 $(\mathrm{n}=9)$, ST421 $(\mathrm{n}=7)$, and ST2288 $(\mathrm{n}=5)$. The most prevalent clusters in 2012 included ST1053 $(n=4)$, ST2318 $(n=4)$, ST5990 $(n=4)$, and ST1614 $(n=4)$. Isolates with identical or phylogenetically similar STs had similar MICs of ceftriaxone. Many novel STs were identified.

Conclusion Ceftriaxone and spectinomycin can continuously be recommended for treatment of gonorrhoea in Nanjing, China. The different molecular epidemiologic clusters in 2007 and 2012 indicate fluctuations in the sexual networks in Nanjing. The identified correlations between NG-MAST STs and MICs of antimicrobials suggest that NG-MAST can supplement the AMR surveillance in China, which needs to be further strengthened.

\section{P3.289 MIC CREEP TO CEFTRIAXONE AND LOW LEVELS OF RESISTANCE TO AZITHROMYCIN IN 7 COUNTRIES FROM SOUTH AMERICA AND THE CARIBBEAN}

doi:10.1136/sextrans-2013-051184.0744

The Gonococcal Antimicrobial Susceptibility Program (GASP) Network in Latin Americathe Caribbea, ${ }^{1 P}$ Araya, ${ }^{2} \mathrm{G}$ Borthagaray, ${ }^{3 P}$ Galarza, ${ }^{4} \mathrm{~A}$ Llop, ${ }^{5} \mathrm{D}$ Payares, ${ }^{6} \mathrm{O}$ M Sanabria, ' $\mathrm{S} D$ Thakur, ${ }^{8} \mathrm{M}$ E Trigoso, 9 J R Dillon. ${ }^{1 / n}$ stituto de Salud Publica de Chile, Santiago, Chile, ${ }^{2}$ Faculdad de Quimica, Universidad de la Republica, Montevideo, Uruguay: ${ }^{3}$ Centro Nacional de Referencia en ITS INEI-ANLIS "Dr Carlos Malbran", Buenos Aires, Argentina; "Instituto de Medicina Tropical "Pedro Kouri", Ministerio Salud Publica, Habana, Cuba; ${ }^{5}$ nstituto Nacional de Higiene "Rafael Rangel", Caracas, Venezuela; 'Instituto Nacional de Salud, Bogota, Colombia; 'GASP-LAC Coordintating Centre, University of Saskatchewan, Saskatoon, SK, Canada; ${ }^{8}$ Centro Departamental de Vigilancia, Informacion y Referencia, CDVIR, La Paz, Bolivia, Plurinational State of; ${ }^{9}$ University of Saskatchewan, Saskatoon, SK, Canada

Background The World Health Organization (WHO) issued an international action plan in 2012 to mitigate the health impact of antimicrobial resistant Neisseria gonorrhoeae isolates. A key strategy is to strengthen international surveillance of gonococcal antimicrobial susceptibility. The Gonococcal Antimicrobial Surveillance Program (GASP) in Latin America and Caribbean (LAC) has reported on AMR trends from 1990. The present study presents regional trends in antimicrobial susceptibility between 2010 and 2011.

Methods Seven countries reported using either agar dilution (CLSI), Etest or disc diffusion assays to determine antimicrobial susceptibility. Countries were asked to report MIC data and categories of susceptibility.

Results Seven countries tested 1019 isolates of $N$. gonorrhoeae in 2010 and 1216 isolates in 2011 to ceftriaxone, penicillin, tetracycline and ciprofloxacin $(n=7)$; azithromycin $(n=4)$ and spectinomycin $(n=3)$. Several countries reported a 2 -fold increase in $\mathrm{MIC}_{50}$ to ceftriaxone (from 0.004 to $0.008 \mu \mathrm{g} / \mathrm{mL}$ ) between 2010 and 2011 and 12 isolates with ceftriaxone MICs $0.125-\geq 0.25 \mu \mathrm{g} / \mathrm{ml}$ were reported in 2011. All isolates were susceptible to spectinomycin. Resistance to azithromycin increased slightly from 1 . $0 \%(6 / 612)$ to $1.7 \%$ (20/1169) while resistance to ciprofloxacin decreased from $42.1 \%$ (429/1019) to $36.2 \%(439 / 1214)$ of isolates tested between 2010 and 2011. Resistance to penicillin increased from 31\% (310/1016) in 2010 to $35 \%$ (428/1216) in 2011 while the percentage of isolates resistant to tetracycline was stable $(2010-21.8 \%, 187 / 858 ; 2011$ $22.6 \%, 275 / 1216)$
Conclusions Third generation cephalosporins and spectinomycin continue to be viable options for the treatment of gonorrhoea in the countries reporting. Low percentages of resistance to azithromycin continue to be reported. There has been a steady decline in capacity for $N$. gonorrhoeae diagnosis and antimicrobial susceptibility testing in the region. The implementation of the WHO action plan to control the spread and impact of antimicrobial resistance in $N$. gonorrhoeae is an urgent priority.

\section{P3.290 HIGH RATES OF CHLAMYDIA POSITIVITY IN ABORIGINAL AND TORRES STRAIT ISLANDER PEOPLE ATTENDING AUSTRALIAN SEXUAL HEALTH SERVICES; THE AUSTRALIAN COLLABORATION FOR CHLAMYDIA ENHANCED SENTINEL SURVEILLANCE (ACCESS)}

doi:10.1136/sextrans-2013-051184.0745

${ }^{1,2,3}$ C C O'Connor, ${ }^{1} \mathrm{H}$ Ali, ${ }^{1} \mathrm{R}$ Guy, ${ }^{4,5} \mathrm{C}$ K Fairley, ${ }^{4,5} \mathrm{M}$ Y Chen, ${ }^{6} \mathrm{~L}$ Marshall, ${ }^{1} \mathrm{~J}$ Kaldor, ${ }^{7} \mathrm{M}$ Hellard, ${ }^{1,8} \mathrm{~B}$ Donovan, ${ }^{1,9} \mathrm{~J}$ Ward. ${ }^{1}$ Kirby Institute, The University of New South UK, Sydney, Australia; ${ }^{2 R P A}$ Sexual Health, Sydney Local Health District, Sydney, Australia; ${ }^{3}$ Central Clinical School, Sydney University, Sydney, Australia; ${ }^{4}$ School of Population Health, University of Melbourne, Melbourne, Australia; ${ }^{5}$ Melbourne Sexual Health Centre, Melbourne, Australia; ${ }^{6}$ Fremantle Hospital, Fremantle, Australia; ${ }^{7}$ Centre for Population Health, Macfarlane Burnett Institute for Medical Research and Public Health, Melbourne, Australia; ${ }^{8}$ Sydney Sexual Health Centre, Sydney Hospital, Sydney, Australia; ${ }^{\circ}$ Baker Institute, Alice Springs, Australia

Introduction Australia has a widely dispersed network of public sexual health services that see large numbers of people at risk of genital Chlamydia trachomatis infection. ACCESS was established to monitor chlamydia testing and positivity rates nationally and to assist the interpretation of chlamydia diagnoses reported through passive surveillance. We report on chlamydia testing and positivity in Aboriginal and Torres Strait Islander (hereafter Aboriginal) people attending 18 sexual health services participating in ACCESS between 2006 and 2011.

Methods Using line-listed data, we analysed Aboriginal status reporting, testing rates based on first visits and chlamydia positivity in those tested. Outcomes were stratified by age group, sex, and year of attendance and were compared with non-Indigenous clients using a chi-square test and multivariate logistic regression $(p<0.05)$. Results From 2006 to 2011, 7,103 (4.2\%) Aboriginal people and 161,626 (95.8\%) non-Indigenous people attended participating sexual health services for an initial visit. Of the Aboriginal people 5,280 (74\%) were tested for chlamydia. The positivity rates in Aboriginal people were $17.0 \%$ in women $(23.3 \%$ in $15-19$ year olds and $18.9 \%$ in $20-24$ year olds) and $17.3 \%$ in men (20.2\% in $15-19$ year olds and $24.3 \%$ in $20-24$ year olds). There were increasing trends seen in chlamydia positivity in Aboriginal and Torres Strait Islander females and non-Indigenous males and females between 2006 and 2011 ( $p$-trend $<0.01)$. On multivariate analysis, positivity was associated with younger age, being heterosexual and living in Queensland in both Aboriginal men and women. In addition, in Aboriginal men, positivity was associated with not living in a remote area, and not having sex overseas; and in Aboriginal women, it was associated with attending in 2010 or 2011.

Conclusion The high Chlamydia positivity rates and increases over time highlight the need for enhanced prevention and screening programmes in Aboriginal people in Australia.

\section{P3.291 ASSOCIATIONS OF CHLAMYDIA TRACHOMATIS INFECTION IN MEN AND WOMEN WITH GENITAL DISCHARGE SYNDROMES IN JOHANNESBURG, SOUTH AFRICA}

doi:10.1136/sextrans-2013-051184.0746

F Radebe, C Ricketts, V Kekana, A Vezi, I Basson, P Magooa, N Bhojraj-Sewpershad, G de Gita, V Maseko, D A Lewis. National Institute for Communicable Diseases (NHLS), Sandringham, South Africa 
Background As part of an on-going STI microbiological surveillance programme, we investigated associations between Chlamydia trachomatis infection and the demographic, clinical, microbiological and behavioural characteristics of patients presenting with either male urethral discharge syndrome (MUDS) or vaginal discharge syndrome (VDS) to a public healthcare facility in Johannesburg, South Africa

Methods 1,218 MUDS and 1,232 VDS cases were consecutively recruited during 6 annual surveys, starting in 2007. Genital discharge pathogens were detected using a molecular assay for $N$. gonorrhoeae, Chlamydia trachomatis, Trichomonas vaginalis, Mycoplasma genitalium and by microscopy of vaginal smears (bacterial vaginosis, Candida). Serology was used to detect syphilis, HSV-2 and HIV infections. Chi-squared tests and logistic regression analyses were used to identify predictors of $C$. trachomatis infection.

Results Overall, 286 (23.5\%) men and 197 (16.0\%) women were C. trachomatis positive, with the highest prevalence observed in men $(30.5 \%)$ and women $(25.9 \%)$ aged $20-24$ years. In the multivariate analysis, C. trachomatis was less likely to be detected in MUDS patients co-infected with $N$. gonorrhoeae (aOR 0.36, 95\% CI, 0.260.49 ) and HSV-2 (aOR $0.70,95 \%$ CI, 0.51-0.95) as well as patients who had received antibiotics recently (aOR 0.43 . 95\% CI, 0.20 0.91). In contrast, the likelihood of $C$. trachomatis infection was higher in VDS patients co-infected with either $N$. gonorrhoeae (aOR 2.22 , 95\% CI, 1.48-3.32) or M. genitalium (aOR 2.24, 95\% CI, 1.45 3.47 ) infection but lower in women who were older (aOR $0.68,95 \%$ CI $0.60-0.77$ ) or who had Candida morphotypes detected (aOR $0.66,95 \%$ CI $0.44-0.98)$.

Conclusion The increased likelihood of co-existent gonococcal or $M$. genitalium infections, but the decreased likelihood of Candida infection, in women with VDS-associated chlamydial infection suggests that these women are at higher risk of STIs, and potentially

HIV. Paradoxically, gonococcal infection as well as recent antibiotic use, reduced the likelihood of MUDS patients having chlamydial infection.

\section{P3.292 THE 2013 IUSTI EUROPEAN COLLABORATIVE CLINICAL GROUP (ECCG) REPORT ON THE DIAGNOSIS AND MANAGEMENT OF CHLAMYDIAL INFECTIONS IN EUROPE}

doi:10.1136/sextrans-2013-051184.0747

B Brooks, ${ }^{1} \mathrm{~J}$ Banks, ${ }^{2} \mathrm{C}$ Ison, ${ }^{3} \mathrm{M}$ van de Laar, ${ }^{4} \mathrm{H}$ Moi, ${ }^{5} \mathrm{~K}$ Radcliffe, ${ }^{6} \mathrm{M}$ Unemo, ${ }^{5} \mathrm{~J}$ Ross, ${ }^{7} \mathrm{~J}$ White, 'R Patel. 'University of Southampton, Southampton, UK; ${ }^{2}$ Health Protection Agency, London, UK; ${ }^{3}$ European Centre for Disease Prevention and Control, Stockholm, Sweden; ${ }^{4}$ University of Oslo, Oslo, Norway; ${ }^{5}$ University Hospitals Birmingham, Birmingham, UK; ${ }^{6}$ World Health Organization Collaborating Centre for Gonorrhoea and other Sexually Transmitted Infections, Örebro University Hospital, Örebro, Sweden; ${ }^{7}$ St Thomas' Hospital London, London, UK

Background The European Collaborative Clinical Group (ECCG) is an expanding network of over 120 Sexually Transmitted Infection specialists from 36 European countries, who collaborate to conduct questionnaire based research across the European Region to identify variations in practise and inform development of international evidence-based guidelines for diagnosis and management of STIs.

The use of sensitive and specific assays and widespread screening has identified clearly the substantial burden of chlamydial infections across Europe. Infection remains common, despite established screening and treatment programmes in many European countries. Recent data has recently challenged the effectiveness of opportunistic screening and standard short course azithromycin therapy and guidance on tests of cure and partner management remain controversial.

Method The 2013 ECCG survey focuses on the diagnosis and management of chlamydial infection. An online survey constructed around clinical scenarios is gathering data on the type of test, site of testing, use of sample pooling and treatment choices. Follow up, including tests of cure and partner management is also being assessed.

Results Interim analysis of results to date show considerable variation in most of the areas studied. Despite their clear advantage many European populations are still denied the benefits of NAATs based testing. Importantly first line antibiotic choice varies as do test pooling strategies and timing and frequency of tests of cure. The survey is currently running and complete data will be available in late spring for full reporting at the conference.

Conclusion As in previous successful ECCG surveys the 2013 ECCG survey on the diagnosis and management of chlamydial infections includes a particular focus on areas where international guidance is currently lacking or poorly detailed. The ECCG has also recently expanded into parts of Eastern Europe and will be able to present data on STI care from this area for the first time.

\section{P3.293 CONDYDAV: PROSPECTIVE OBSERVATIONAL STUDY OF PATIENTS WITH EXTERNAL GENITAL WARTS(EGWS) CONSULTING IN STI CLINICS IN FRANCE}

doi:10.1136/sextrans-2013-051184.0748

IJ Chanal, ${ }^{2} \mathrm{~S}$ Fouéré, ${ }^{3} \mathrm{~F}$ Yassir Oria, ${ }^{4} \mathrm{~N}$ Spenatto, ${ }^{5} \mathrm{~F}$ Bouscarat, ${ }^{6} \mathrm{E}$ Picot, ${ }^{10} \mathrm{Aynaud}$, ${ }^{2} \mathrm{M}$ Janier, 'N Dupin, the groupe MST-Sida from the French Society of Dermatology. ${ }^{1}$ Department of Dermatology, pavillon Tarnier, Hôpital Cochin, APHP, Paris, France; ${ }^{2}$ STI centre, Hôpital Saint Louis, Paris, France; ${ }^{3}$ CIDDIST, Hôpital de la Croix Rousse, Lyon, France; ${ }^{4}$ STI centre, Hôpital de la Grave, Toulouse, France; ${ }^{5}$ CIDDIST, Hôpital Bichat, Paris, France; ${ }^{6}$ Department of Dermatology, Hôpital Saint Eloi, Montpellier, France

In this prospective, observational study, we analysed clinical features and treatments between January, 12011 and March, 312011 of patients consulting for EGWs in 15 STI clinics through France. 372 males and 111 women were included; mean age 31.2 years old. Women were younger than men $(31.7$ and 28.9 y.o respectively $\mathrm{p}<0.05) .414(85.7 \%)$ were heterosexuals, 13 bisexuals and 54 (11.2\%) homosexuals; mean age at first sexual intercourse: 17 y.o. Males reported more sex partners in the last 12 months (more than 3 partners in $32.6 \%$ versus $11.9 \% p<0.01$ ). In males, 230 had an involvement of the penis alone and 46 had an involvement of the anus alone. 76 patients had EGWs of the anus, among them 26 were MSM. In females, 76 had an infection of the vulva alone and 22 a co-infection vulva and anus. Concerning cervical cancer screening, 13 had never been tested (mean age 24.8 y.o. [17-40]); mean delay for the last screening: 12 months [1-108]. Three women have been vaccinated against HPV. 3 males were newly diagnosed HIV positives and 8 for syphilis (7 were MSM RR: 50). In females, 2 patients were HIV positives and $13 \%$ had an infection by Chlamydia trachomatis (11/84 not known: 27). Women were significantly more treated with Imiquimod (35\% Vs $19.9 \%$ p < 0.001).

\section{P3.294 ASSOCIATION OF MYCOPLASMA GENITALIUM WITH CERVICITIS IN NORTH INDIAN WOMEN ATTENDING GYNECOLOGIC CLINICS}

doi:10.1136/sextrans-2013-051184.0749

'S Sethi, ${ }^{2} \mathrm{~N}$ Rajkumari, ${ }^{3} \mathrm{~L}$ Dhaliwal, ${ }^{1}$ A Roy. 'Deptt of Microbiology, Post Graduate Institute of Medical Education and Research, Chandigarh, India; ${ }^{2}$ PGIMER, Chandigarh, India; ${ }^{3}$ Deptt of Obstetrics and Gynaecology, PGIMER, Chandigarh, India

Background The aetiology of mucopurulent cervicitis is not known in $50 \%$ of cases and cannot be attributed to the known cervical pathogens Neisseria gonorrhoeae, Chlamydia trachomatis, Genital Mycoplasmas are such organisms which can be easily overlooked as there is a lack of sufficient laboratory infrastructure for diagnosing them in developing countries. Also, there is the lack of awareness among physicians about $M$. genitalium infection. There is scarce literature showing the association of $\mathrm{M}$. genitalium with cervicitis and no reports from India. The purpose of this study was hence to 